\title{
Accurate characterization of next-generation thin-film photodetectors
}

\author{
The performance of photodetectors fabricated from emerging semiconductors such as perovskites, quantum dots, \\ two-dimensional materials or organics, for example, can be prone to misinterpretation. This Comment exposes the \\ problems and proposes some guidelines for accurate characterization.
}

Yanjun Fang, Ardalan Armin, Paul Meredith and Jinsong Huang

$\mathrm{N}$ ext-generation semiconductors, such as the organohalide perovskites, inorganic nanocrystals and organic semiconductors, are progressively enabling new thin-film optoelectronics, including photovoltaics, light-emitting devices and photodetectors. The basic electro-optical physics of these materials and the related devices are quite different to traditional crystalline systems such as silicon and compound semiconductors. In particular, charge traps and disorder play substantial roles and have a profound influence on electronic properties. This behaviour poses new challenges at the basic materials and device design level - and also means that well-established principles and methods for device characterization are prone to misinterpretation at best, or complete failure at worst. This has led to several unrealistic and potentially erroneous claims of record-high performance metrics in the literature, notably specific detectivities and noise equivalent power (NEP). In this Comment, we address this question for the case of nextgeneration thin-film photodetectors. This is a rapidly expanding field with considerable technological potential but requiring firm performance metric guidelines to establish valid comparisons between different semiconductors and architectures.

\section{Basic performance metrics}

The most basic performance metric for any 'sensor' (a photodetector is a light sensor) is its sensitivity - intuitively the input-output signal efficiency relative to the output noise. For a photodetector, this metric has traditionally been exemplified by theNEP. The NEP is the incident light power at which the signal-to-noise ratio (SNR) is unity and it is related to the responsivity $\left(R_{\text {res }}\right)$ and the noise current $\left(i_{n}\right)$ by equation (1)

$$
\begin{gathered}
\mathrm{NEP}=i_{\mathrm{n}} / R_{\text {res }} \\
R_{\text {res }}=I_{\mathrm{ph}} / P
\end{gathered}
$$

The responsivity (equation (2)) is the photodetector input-output efficiency or the ratio of the output photocurrent $\left(I_{\mathrm{ph}}\right)$ and input light power $(P)$. The inverse of the NEP normalized to the square root of active area is the specific detectivity $\left(D^{*}\right.$ in $\mathrm{cm}$ $\mathrm{Hz}^{1 / 2} \mathrm{~W}^{-1}$ or Jones), which can be thought of as the SNR generated by a photodetector with an active area $(A)$ of $1 \mathrm{~cm}^{2}$ at $1 \mathrm{~W}$ of incident power when the electrical bandwidth $(\Delta f)$ is $1 \mathrm{~Hz}$ :

$$
D^{*}=\frac{\sqrt{A \Delta f}}{\mathrm{NEP}}
$$

While equations (1)-(3) appear to provide a fairly simple 'universal' platform for photodetector performance assessment, they also contain sometimes unappreciated subtleties: notably, the critical role of the noise spectrum, and the incident light intensity and modulation frequency dependence of the responsivity. We will now examine these and comment on their impact in the reported literature as seemingly more and more impressive $D^{*}$ values are reported.

\section{What is noise?}

"While we can describe the physical consequences of order parameter fluctuations in intricate detail, we have comparatively little knowledge about the microscopic origins of voltage fluctuations in a simple resistor." This nearly 40 -yearsold statement of Dutta and Horn ${ }^{1}$ is undoubtedly still pertinent today and particularly so in next-generation photodetectors made from disordered semiconductors. It is nigh impossible to accurately predict the noise current of an arbitrary photodetector from simple models. In this regard, a significant fraction of published papers on thin-film photodetectors assume the noise power spectrum to be 'white', that is, frequency independent, and calculated from the root mean square of the shot noise (due to the uncorrelated arrival of the electrons) and thermal Johnson noise (due to fluctuations of electron density in the circuit):

$$
\left\langle i_{\mathrm{n}}^{2}\right\rangle_{\text {unrealistic }}=\left[2 q\left\langle i_{\mathrm{d}}\right\rangle+\frac{4 k_{\mathrm{B}} T}{R_{\text {shunt }}}\right] \Delta f
$$

where $q$ is the elementary charge, $\left\langle i_{\mathrm{d}}\right\rangle$ is the average dark current, $k_{\mathrm{B}}$ is the Boltzmann constant, $T$ is the temperature and $R_{\text {shunt }}$ the shunt resistance. The attractiveness of this approach is clear: the direct current dark noise as a function of bias and shunt resistance are relatively simple to measure. It is also widespread practice to go even further and ignore the thermal noise, especially at small reverse bias voltages wholly inappropriate especially for 'leaky' photodetectors where there may be substantial defects giving rise to the thermal noise. Irrespective of whether both shot and thermal components are considered, there may be other system-specific mechanisms contributing to the noise current such as flicker or pink $(1 / f)$ noise, or elements of static and dynamic disorder (trapping/ de-trapping, generation/recombination and so on), which both have pronounced frequency dependencies (discussed below). They may dominate the total device noise, especially at low frequencies. The impact of underestimating the noise current is clear from equations (1) and (2): $D^{*}$ is inflated and intensity- and frequency-dependent considerations are swept under the metaphorical carpet.

\section{Frequency dependence}

The responsivity of any photodetector tends to zero when the modulation frequency of the input light exceeds the response speed, which is defined by the resistancecapacitance time, carrier transit time and/or charge trapping release time ${ }^{2}$. The response bandwidth is often considered to be the frequency at which the responsivity drops to $-3 \mathrm{~dB}$ of its low-frequency value. 


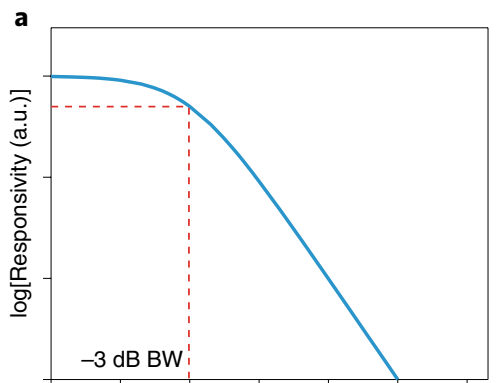

$\log [$ Frequency (a.u.)]

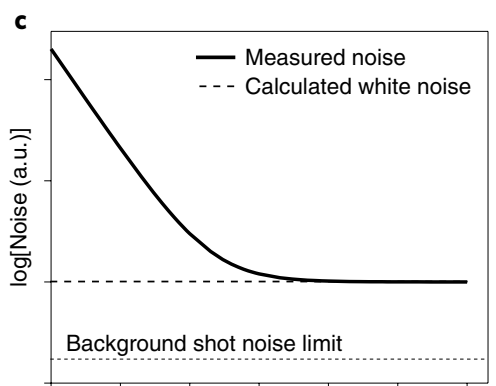

$\log [$ Frequency (a.u.)]

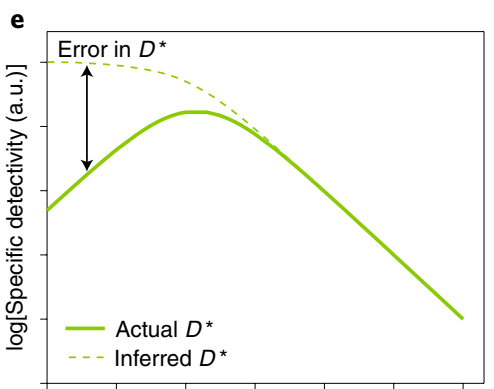

$\log [$ Frequency (a.u.)]

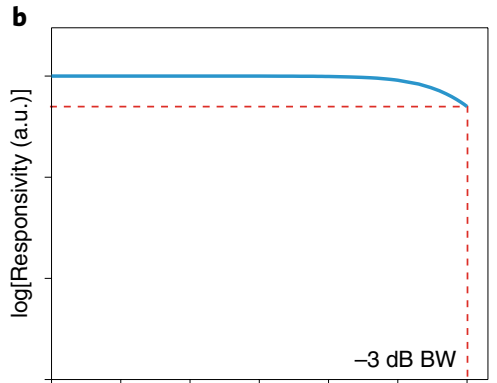

$\log [$ Frequency (a.u.)]

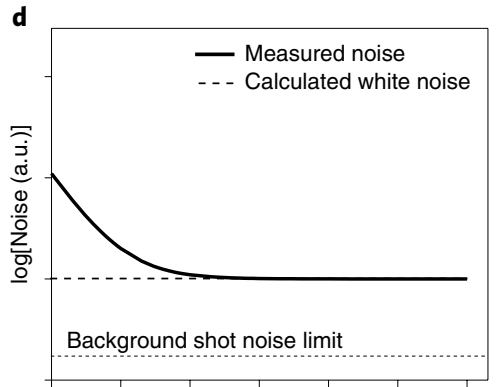

$\log [$ Frequency (a.u.)]

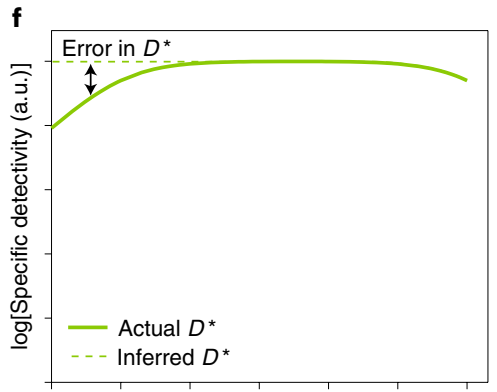

$\log [$ Frequency (a.u.)]

Fig. 1 | Frequency dependence of the responsivity, noise and specific detectivity. a-f, Responsivity

$(\mathbf{a}, \mathbf{b})$, noise spectral density $(\mathbf{c}, \mathbf{d})$ and $D^{\star}(\mathbf{e}, \mathbf{f})$ for two hypothetical photodetectors with strong (left column) and weak (right column) overlap of the $-3 \mathrm{~dB}$ bandwidth (BW) with the flicker noise regime. The red dashed lines in $\mathbf{a}$ and $\mathbf{b}$ indicate the $-3 \mathrm{~dB} B W$ range. The resultant $D^{*}$ calculated from equation (1) (ignoring the flicker noise) and measured noise (equation (2), including flicker noise) can be orders of magnitude different.

The question of whether the noise current is white inside the bandwidth is critical in determining $D^{*}$. In the absence of any system-specific additional noise mechanism, a more realistic version of equation (4) contains the flicker $(1 / f)$ noise spectral power density $\left(i_{1 / f}^{2}(\omega)\right)$, and hence the noise power is frequency $(\omega)$ dependent:

$$
\begin{aligned}
& \left\langle i_{\mathrm{n}}^{2}(\omega)\right\rangle_{\text {realistic }} \\
& \quad=\left[2 q\left\langle i_{\mathrm{d}}\right\rangle+\frac{4 k_{\mathrm{B}} T}{R_{\text {shunt }}}+i_{1 / f}^{2}(\omega)\right] \Delta f
\end{aligned}
$$

Figure 1 shows two cases: the $-3 \mathrm{~dB}$ bandwidth inside (left column) and outside (right column) the flicker regime. In the former case, there is a very significant error in $D^{*}$ if simply inferred from the noise from equation (4) (or indeed worst from only the shot noise). This frequency dependence has been experimentally demonstrated by Konstantanos et al. ${ }^{3,4}$ for $\mathrm{PbS}$ nanocrystal photodetectors with photoconductive gain and narrow frequency bandwidths of $<10 \mathrm{~Hz}$. Thus, once again, we see the need to fully consider the frequency dependence of the noise current and responsivity. Failing that, it is important to clearly state the frequency at which a $D^{\star}$ has been determined. This is of critical importance in devices with photoconductive gain.

\section{Nonlinearity of responsivity}

According to equation (3), $D^{*}$ can be evaluated directly from the NEP. It is widespread practice to determine the NEP from the intensity-dependent photocurrent measured over an experimentally convenient range ( $\mu \mathrm{W}$ to $\mathrm{mW}$ from a monochromator or attenuated laser or light-emitting diode) at a specified wavelength and fixed modulation frequency (or continuous wave) of the incident light. The linear dynamic range (LDR) is another very important photodetector metric and is defined in $\mathrm{dB}$ as the range of linear responsivity, or the range that the responsivity remains constant under different light intensities. The NEP as shown in Fig. 2 can be inferred by extrapolating the photocurrent into the low-intensity limit to the 'chosen' noise current (often the inferred noise from the dark current as described above). This procedure has an inherent assumption that the responsivity is linear as a function of input light intensity all the way to the NEP. While this may be true (although not trivially so) in photodetectors with low trapstate densities and with slow responses, there are clear examples in the literature where the photocurrent decays superlinearly ${ }^{5}$ or sublinearly ${ }^{3}$ at low intensities. Both these circumstances are illustrated in Fig. 2a, and it is clear they could lead to inaccurate estimates of the NEP possibly by orders of magnitude if such a nonlinearity was not understood and accounted for. It is also worth noting that deviation from linearity at high intensities is an additional potential source of extrapolation error - although this is now better understood as the onset of bimolecular recombination or the impact of high series resistance including non-ohmic contacts ${ }^{6}$, and is thus more straightforward to deal with even in systems with gain ${ }^{3,4}$. It should be noted a device with a large photoconductive gain at moderate light intensities does not necessarily indicate a large $D^{*}$ or superior low-light detection capability due to possible charge-trapping effects when the incident light intensity approaches the NEP. This again highlights the importance of measuring the LDR directly. Moreover, one should avoid confusing optical power with optical power density when reporting the NEP. This may be particularly problematic for the case of nanomaterial-based photodetectors with small device areas where, although the power detection limit may be very low, the determined NEP is not based on the correct power density analysis. For example, in single nanowire-based photodetectors whose radial dimensions may be below the wavelength of light being measured, the determination of an 'effective device area' is difficult when taking into account factors such as the polarization of the incoming photon with respect to the nanowire orientation, and also the diffraction of light. This could lead to large uncertainties in the reported NEP and $D^{\star}$. Finally, the 


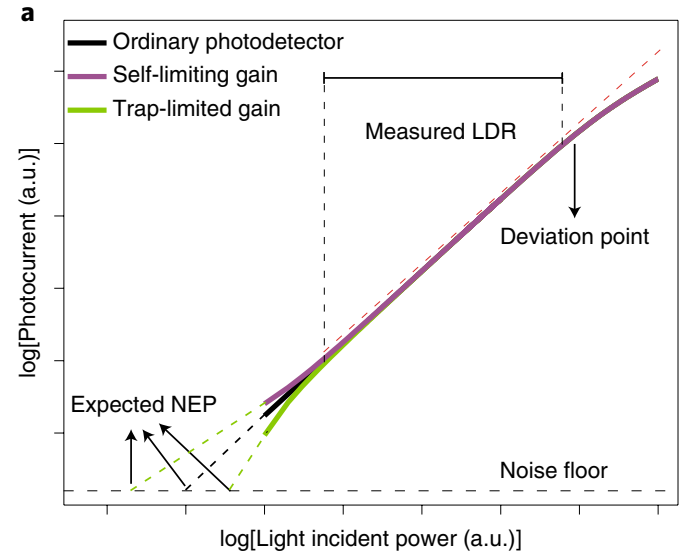

b

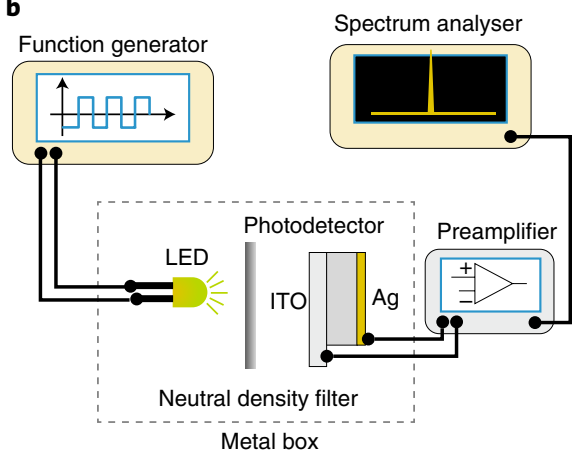

Fig. 2 | Noise equivalent power determination. a, Intensity-dependent photocurrent measurements showing the potential nonlinearity of the responsivity at low intensities for two cases: self-limiting gain with superlinear behaviour ${ }^{3}$; and trap-limited gain with sublinear behaviour ${ }^{5}$. In both cases, linear extrapolation based on the LDR region delivers incorrect estimates of the NEP. The red dashed line represents the linear response of photocurrent with light incident power. $\mathbf{b}$, Set-up for frequencydependent measurement of the current spectral density. $\mathbf{c}$, Measurement result for a hypothetical photodetector showing the noise floor and single modulation frequency $(1 \mathrm{~Hz}$ bandwidth) photoresponse with varying incident light intensity. Inset shows the SNR derived from this frequency-dependent current, which can be used to accurately determine the NEP. LED, light-emitting diode; ITO, indium tin oxide.

LDR should be determined from the double logarithmic plot where the slope is unity. There are several examples in the literature where the 'LDR' is erroneously evaluated from double logarithmic plots of photocurrent versus light intensity with non-unity slope.

\section{Measurement protocols}

From the above discussion, it is clear that an 'inferred' evaluation of $D^{*}$ derived from the thermal and the shot noise is simply not adequate. Ideally, $D^{\star}$ should be derived from a careful noise spectral density measurement obtained over the frequency and bias range of relevance for the photodetector application and performance window. This can be done by applying a fast Fourier transform to the current versus time ${ }^{6-9}$, or direct measurement via a fast Fourier transform or swept super-heterodyne spectrum analyser ${ }^{10-12}$. In addition, a lock-in

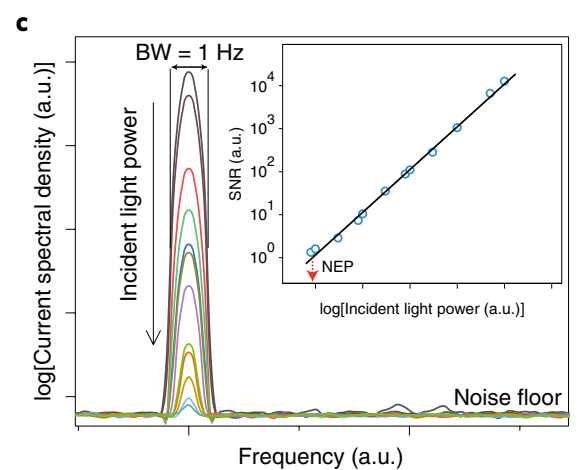

amplifier can be used to measure the noise at each reference frequency and at a given bandwidth (time constant); noting it is essential that the magnitude $(R)$ is measured or the in-phase component when the phase difference between the signal and (dummy) reference is set to zero. This is particularly important for high $D^{\star}$ claims $>10^{11}$ Jones, and certainly claims $>10^{13}$ Jones (difficult to even measure) require considerable justification and cross-checking, even more so when photoconductive gain is invoked. One possible approach to verify $D^{\star}$ is to independently and directly determine the NEP. As shown in Fig. 2a and described above, this can be facilitated by the accurate measurement of the LDR over the broadest possible intensity range (and under relevant bias and modulation frequency conditions), noting both the appropriate and correct noise floor and possible deviations from linearity in the low- and high-intensity regimes ${ }^{13-17}$. Accurate reporting of the LDR, $D^{*}$ and frequency bandwidth are essential features of a well-characterized photodetector alongside the bandwidth and response times.

A second method to accurately determine the NEP is to measure the photodetector current spectral density under modulated light at various light intensities ${ }^{10-12}$, as schematically shown in Fig. 2b. As shown in Fig. 2c, the width of the peak is the electrical bandwidth and its magnitude the photoresponse. The SNR can be then plotted as a function of input light power, and the intercept with $\mathrm{SNR}=1$ is an accurate measure of the NEP with no extrapolation when the electrical bandwidth of the measurement (resolution bandwidth of the spectrum analyser) is set to $1 \mathrm{~Hz}$. In other words, the intensity at which the photocurrent peak can no longer be differentiated from the noise floor gives a measure of the NEP. This is a direct methodology by which one can determine the LDR, NEP and $D^{*}$ in one go with no extrapolation needed. Notably, the quantum nature of photons (and the related photon shot noise) should be taken into account to obtain the accurate 'effective NEP or $D^{*}$ ' when the incident or absorbed light power is extremely weak ${ }^{18}$. However, its influence is negligible for photodetectors with $D^{*}<10^{13}$ Jones, whose lowest detectable photon number flux density is generally above $10^{6} \mathrm{~cm}^{-2} \mathrm{~s}^{-1}$, far away from the region that the quantum effects of photons play a role.

Finally, and in common with other optoelectronic device characterization challenges (including solar cells), there are a number of additional pitfalls that need to be avoided and which boil down to good experimental design. These include: accurate calibration of the intensity of light sources, and careful management of stray light (reflected, transmitted or scattered) especially at low intensities; lowering the noise floor of the measurement equipment using low-noise amplifiers with sufficient bandwidth and clean power sources; minimization of pick-up noise using co- or tri-axial cables and Faraday cages; avoidance of over-illumination especially with smallarea devices - it is common practice in solar cells to use illumination masks; accurate measurement of device area so that current and power densities are correct; and care when measuring response times so that they are not limited by external circuit resistance-capacitance, illumination source pulse widths or mechanical chopper turnon-off times ${ }^{11,19}$

In summary, the field of thin-film photodetectors based on next-generation semiconductors is rapidly expanding with 
significant technological potential. Protocols for accurate determination and verification of comparative performance figures of merit for these devices are urgently required, and there are potential examples in the literature $\mathrm{e}^{2,4,6,7,12,19,20}$. The widespread practice of simply defining the specific detectivity from the responsivity and dark or shot noise is wholly inadequate and ignores the subtlety of the frequency and input-lightintensity dependence of all key parameters. This approach can overestimate $D^{*}$ by orders of magnitude. We thus suggest that an accurate and independent NEP be determined via separate measures of the noise spectral density and responsivity as a function of frequency and illumination intensity. This should be a required feature of all new photodetector reports alongside careful LDR and bandwidth analysis. Optimally, a combined noise spectral density and photocurrent measurement at fixed modulation frequency (and $1 \mathrm{~Hz}$ bandwidth) can directly deliver the SNR and NEP. This process is absolutely essential for validating high $D^{*}$ claims and understanding the potential impact of photoconductive gain.

Yanjun Fang ${ }^{1}$, Ardalan Armin², Paul Meredith $^{2 *}$ and Jinsong Huang ${ }^{1 *}$

${ }^{1}$ Department of Applied Physical Sciences, University of North Carolina, Chapel Hill, NC, USA. ${ }^{2}$ Department of Physics, Swansea University, Swansea, UK.

*e-mail:Paul.Meredith@Swansea.ac.uk;

jhuang@unc.edu

Published online: 14 December 2018

https://doi.org/10.1038/s41566-018-0288-z

\section{References}

1. Dutta, P. \& Horn, P. M. Rev. Mod. Phys. 53, 497-516 (1981).

2. García de Arquer, F. P., Armin, A., Meredith, P. \& Sargent, E. H. Nat. Rev. Mater. 2, 16100 (2017).

3. Konstantatos, G., Clifford, J., Levina, L. \& Sargent, E. H. Nat. Photon. 1, 531-534 (2007).

4. Konstantatos, G. et al. Nature 442, 180-183 (2006)

5. Guo, F. et al. Nat. Nanotech. 7, 798-802 (2012).

6. Armin, A. et al. Laser Photon. Rev. 8, 924-932 (2014).
7. Armin, A., Jansen-van Vuuren, R. D., Kopidakis, N., Burn, P. L. \& Meredith, P. Nat. Commun. 6, 6343 (2015).

8. Lopez-Sanchez, O., Lembke, D., Kayci, M., Radenovic, A. \& Kis, A. Nat. Nanotech. 8, 497-501 (2013).

9. Liu, C.-H., Chang, Y.-C., Norris, T. B. \& Zhong, Z. Nat. Nanotech. 9, 273-278 (2014)

10. Dong, R. et al. Adv. Mater. 27, 1912-1918 (2015)

11. Fang, Y. \& Huang, J. Adv. Mater. 27, 2804-2810 (2015).

12. Zhang, L. et al. Adv. Mater. 27, 6496-6503 (2015).

13. Guo, F., Xiao, Z. \& Huang, J. Adv. Opt. Mater. 1, 289-294 (2013).

14. Fang, Y., Guo, F., Xiao, Z. \& Huang, J. Adv. Opt. Mater. 2, 348-353 (2014).

15. Bao, C. et al. Adv. Mater. 29, 1703209 (2017).

16. Zhang, Y., Yuan, Y. \& Huang, J. Adv. Mater. 29, 1603969 (2017).

17. Yuan, Y. \& Huang, J. Adv. Opt. Mater. 4, 264-270 (2016).

18. Rose, A. Vision Human and Electronic Ch. 1 (Plenum Press, New York, 1973).

19. Shen, L. et al. Adv. Mater. 28, 10794-10800 (2016).

20. Lin, Q., Armin, A., Burn, P. L. \& Meredith, P. Nat. Photon. 9 687-694 (2015)

\section{Acknowledgements}

J.H. and Y.F. acknowledge financial support from the Academic Research Initiative in Department of Home Security under award 2014-DN-077-ARI069 and the National Science Foundation under award ECCS -

1747674. P.M. and A.A. acknowledge the support of the European Regional Development Fund (Welsh European Funding Office) and Swansea University for the Sustainable Advanced Materials strategic initiative. 